\title{
Tackling Health Inequalities Using Urban HEART in the Sustainable Development Goals Era
}

\author{
Amit Prasad (D) C Carme Borrell • \\ Roshanak Mehdipanah • Somnath Chatterji
}

Published online: 20 April 2018

(C) The New York Academy of Medicine 2018

In 2015, more than half of the world's population lived in cities (54.6\%), and this percentage is expected to increase to $67 \%$ by 2050 . Given this growth, the processes occurring in urban areas are important to understanding the economic, social, political, and health transformations in a country. Studies have revealed an urban advantage for health in comparison to rural areas, both in terms of access to services and for many health outcomes. Greater economic resources, higher density, and better infrastructure and service availability contribute to the potential for good health in cities [1].

However, while cities possess resources in abundance, these resources are often concentrated, especially in low- and middle-income countries (LMICs), in the hands of a relatively small proportion of the population and the presumed urban advantage systematically excludes hundreds of millions of people in cities, globally. This results in inequalities not only in the

\footnotetext{
A. Prasad $(\bowtie) \cdot$ S. Chatterji

Department of Information, Evidence and Research, World Health Organization, Geneva, Switzerland

e-mail: prasada@who.int

C. Borrell

Agència de Salut Pública de Barcelona, Barcelona, Spain

C. Borrell

CIBER of Epidemiology and Public Health, Madrid, Spain

R. Mehdipanah

Department of Health Behavior and Health Education, University of Michigan School of Public Health, Ann Arbor, MI, USA
}

socioeconomic domain but also in access to health services and health outcomes [2].

Inequities in health concern systematic inequalities in health status between different social groups. In addition to being systematic, these differences are socially produced and are, therefore, unjust and modifiable [3]. In urban areas, socioeconomic inequalities in health tend to be larger among populations that are disadvantaged, poor, and concentrated in marginalized neighborhoods, usually in the suburb outskirts of the city or in inner city areas. These areas also tend to have a higher incidence of drug overdose, tuberculosis, and HIV/AIDS [4-6].

Cities in LMICs, in particular, face severe problems in distributing limited resources among rapidly growing urban populations. This mismatch of population increases and limited resources is most visibly manifested in the proliferation of slums and informal settlements. More than 880 million people are currently estimated to live in slum conditions around the world. Poor housing conditions, overcrowding, lack of access to safe water and sanitation, and a lack of secure tenure characterize slums. They account for about three in five people in urban areas of sub-Saharan Africa, one in three people in urban Asia and one in five people in urban Latin America and the Caribbean. Projections reveal that this is expected to more than double to two billion people by $2050[1,7]$.

The Urban Health Equity Assessment and Response Tool (Urban HEART) was developed jointly by the World Health Organization (WHO), city and national officials, and academics and researchers in 2010 to support stakeholders in cities to address health inequities by acting on the social, economic, and physical 
environmental determinants of health [8]. More than 100 cities in 53 countries had used the tool to plan actions on health inequities by the end of 2015. Urban HEART guides users through a process of bringing different stakeholders together, collecting and collectively analyzing disaggregated data on health and its determinants, and planning action to reduce inequities informed by best practices. The tool has been used in diverse environments, from small cities such as Matsapha, Swaziland, with a population of 35,000 to Tehran, the Islamic Republic of Iran, home to more than eight million people. Cities in countries at varying levels of development such as Canada and Mali have used Urban HEART to achieve the same goal of reducing health inequities. The tool has also been independently evaluated in 15 cities from seven countries [1].

In September 2015, 193 Member States of the United Nations adopted a new set of 17 global development goals with 169 national targets called the Sustainable Development Goals (SDGs) for 2016-2030. The SDGs cover a wide range of development issues such as ending poverty, achieving universal education and combating climate change. Improving health will remains a global priority during 2016-2030 with SDG 3 focused on ensuring healthy lives and promoting well-being for all at all ages. Another SDG (SDG 11) is dedicated to making cities inclusive, safe, sustainable, and resilient. Keys to achieving this goal include housing, air quality, and transport, which are also important determinants of people's health in cities. The SDGs offer a new platform for cities to connect globally and cooperate on a wide range of issues, including health, environment, and the economy [9].

While Urban HEART was originally aligned to monitor the achievement of the Millennium Development Goals (MDGs) at the local level, the tool has the potential to support stakeholders in cities to monitor SDGs for health and the determinants of health with a focus on equitable progress [10]. There are three fundamental characteristics of Urban HEART that make it suitable for doing so. First, the framework for Urban HEART is derived from the work of the Commission on Social Determinants of Health (CSDH). The CSDH highlighted the imperative to address the wider social, economic, environmental, and political determinants in order to reduce health inequities [11]. The Urban HEART framework for categorizing indicators and interventions includes health outcomes and four policy domains on the social determinants of health. These four policy domains are physical environment and infrastructure, social and human development, economics, and governance. Indicators currently included in these domains span the breadth of issues covered by the SDGs, except for the goal on "Life Below Water." Cities have added and aligned indicators in accordance with their specific social, economic, and political context such as in Barcelona, Detroit, and Manila. These adaptations serve as an important insight into local priorities that can enrich the universe of indicators available to future users of the tool.

Second, the emphasis on an inclusive approach to development in the SDGs is strongly captured not only with the goal of health equity but also within the process of Urban HEART. Three integral processes to the implementation of Urban HEART are the collection and use of sound evidence, intersectoral action for health, and community participation. Social exclusion is a key determinant of health inequities in cities as some population groups such as those living in slums are systematically excluded from participating in decision-making processes. The Urban HEART process has sought to remedy this issue by engaging affected communities both in the identification of issues and in the development and implementation of actions, illustrated by the experiences in Detroit and Tehran.

Third, recent advancements in information and communication technologies (ICTs) will ensure synergies between SDG monitoring and Urban HEART. Taking into account that it is difficult to have intra-city data, generation of large scale data, termed as "big data," can help overcome the lack of quality data at the local level. The advent of big data has ushered in a data revolution that can potentially strengthen national and local health information systems and provide better decision-support tools for policy-makers [12]. It can also support monitoring of the impact of interventions in real time both at the individual and the public heath level and guide implementation research to ensure that policies have their desired impact. This is especially important with the rise in prevalence of noncommunicable diseases and mental health in cities. A critical advantage of big data is that it has the potential to democratize information and engage the public in transparent decision-making processes. Urban HEART, with its focus on social determinants of health and a participatory approach, is well suited to take advantage of contemporary developments in data collection.

This series aims to demonstrate the scope of actions undertaken to reduce health inequities using Urban 
HEART in different political, economic, social, and geographically diverse contexts. The four papers following this are focused on the experiences of Barcelona, Detroit, Matsapha, and Tehran. They convey the scope and substance of implementation, with each city facing some common as well as unique challenges relating to their political context, funding, technical issues, and social environment. In Matsapha, a low-resourced setting, the availability of disaggregated data at the town level is a major barrier but is overcome by combining information from different programs and a joint effort by stakeholders to supplement required data. Community-based participatory process adopted in Detroit contributed to benchmark selection and modification and provided opportunities for dialogue and colearning throughout the process. The Barcelona experience emphasizes the role of a strong political will in order to place health inequalities in the political agenda and support the implementation of tools like Urban HEART to tackle them.

"No one must be left behind" is the refrain of the 2030 Agenda for Sustainable Development. As cities will account for $60 \%$ of the world's population by 2030 , progress in urban health will be critical to achieving the SDGs. Urban HEART will be particularly important with respect to achieving Universal Health Coverage (UHC), a core health SDG, and improving the distribution of the social determinants of health to ensure that the most vulnerable and difficult to reach populations in cities get high quality interventions they need without having to incur financial hardship. It has the potential to increase awareness of the need to improve health equity, support the adoption of a participatory evidence-based approach to deliver appropriate prevention and promotion strategies, and ensure adherence to treatments and assist with structural changes.

\section{References}

1. WHO, UN Habitat. The global report on urban health: equitable, healthier cities for sustainable development. Geneva, Switzerland: World Health Organization; 2016.

2. UN Habitat. State of the world's cities 2010/2011-cities for all: bridging the urban divide. Nairobi, Kenya: United Nations Human Settlements Programme; 2010.

3. Whitehead M, Dahlgren G. Concepts and principles for tackling social inequities in health. Levelling up (part 1). World Health Organization: studies on social and economic determinants of population health $n^{\circ} 2$, Denmark, Copenhagen: WHO Europe; 2006.

4. Marí-Dell'Olmo M, Gotsens M, Palència L, et al. Trends in socioeconomic inequalities in mortality in small areas of 33 Spanish cities. BMC Public Health. 2016;16:663. https://doi.org/10.1186/s12889-016-3190-y.

5. Diez Roux AV, Green Franklin T, Alazraqui M, Spinelli H. Intraurban variations in adult mortality in a large Latin American city. J Urban Health. 2007;84(3):319-33.

6. Karpati A, Kerker B, Mostashari F, et al. Health disparities in New York City. New York: New York City Department of Health and Mental Hygiene; 2004.

7. WHO, UN Habitat. Hidden cities: unmasking and overcoming health inequities in urban settings. Geneva, Switzerland: World Health Organization; 2010.

8. WHO. Urban Health Equity Assessment and Response Tool (Urban HEART). Kobe, Japan: WHO Centre for Health Development; 2010.

9. United Nations. Website: http://www.un. org/sustainabledevelopment/sustainable-developmentgoals. [last accessed 28 March 2017].

10. Prasad A, Kano M, Dagg K, et al. Prioritizing action on health inequities in cities: an evaluation of Urban Health Equity Assessment and Response Tool (Urban HEART) in 15 cities from Asia and Africa. Soc Sci Med. 2015;145:237-42.

11. Marmot M, Friel S, Bell R, Houweling TA, Taylor S. Commission on Social Determinants of Health. Closing the gap in a generation: health equity through action on the social determinants of health. Lancet. 2008;372(9650):1661-9.

12. Andreu-Perez J, Poon CCY, Merrifield RD, et al. Big data for health. IEEE Journal of Biomedical and Health Informatics. 2015;19(4) 